\title{
Nonproportional Correlative Reduction Finite Element Method for Slope Strength Parameters
}

\author{
Haibin Xue, ${ }^{1}$ Faning Dang, ${ }^{1}$ Xiaotao Yin, ${ }^{2}$ Weihua Ding, ${ }^{1}$ and Chao Yang ${ }^{1}$ \\ ${ }^{1}$ Institute of Geotechnical Engineering, Xian University of Technology, Xian 710048, China \\ ${ }^{2}$ Institute of Rock and Soil Mechanics, Chinese Academy of Sciences, Wuhan 430071, China \\ Correspondence should be addressed to Haibin Xue; xhbyts2013@163.com
}

Received 5 August 2016; Accepted 10 October 2016

Academic Editor: Edoardo Artioli

Copyright (c) 2016 Haibin Xue et al. This is an open access article distributed under the Creative Commons Attribution License, which permits unrestricted use, distribution, and reproduction in any medium, provided the original work is properly cited.

\begin{abstract}
To solve the problems presented by the rational determination of the reduction mode in the traditional strength reduction method and to overcome the difficulty in determining double safety factors from the existing double reduction factors method, a nonproportional relationship between the cohesion reduction factor and the internal friction angle reduction factor is established for a case in which the strength parameter distribution obeys the linear attenuation assumption. By introducing this correlation into the traditional strength reduction finite element method, the nonproportional correlative reduction finite element method for slope strength parameters is proposed. To verify the reliability of the proposed method, the nonproportional correlation is introduced into Bishop's slice method, yielding the nonproportional double safety factors of Bishop's slice method. For the whole safety storage of the slope based on the double safety factors, a comprehensive safety factor with the contribution of the shear strength parameter to the sliding resistance force as weight is proposed. Finally, in combination with an example and a comparative analysis of the slope sliding surface positions and the comprehensive safety factors for three different types of reduction modes with two types of methods, the rationality and reliability of this method were validated.
\end{abstract}

\section{Introduction}

The strength reduction finite element method provides incomparable advantages over the limit equilibrium method. When the sliding surface shape and position do not need to be assumed in advance, the strength storage safety factor for slopes can be directly obtained. Accordingly, with the rapid development of the computing technology, this method has been extensively used for analyzing the slope stability.

Since 1975, when Zienkiewicz et al. [1] proposed the strength reduction finite element method, many scholars have performed a considerable number of studies in this research area. For example, Ugai [2], Matsui and San [3], Song [4], Griffiths and Lane [5], Dawson et al. [6], Liu et al. [7], Zheng and Liu [8], and Wu et al. [9] have conducted research on slope failure criteria. Zhang et al. [10] and Fu and Liao [11] have studied the effects of different yield criteria on the slope plastic zone and the strength reduction safety factor. Manzari and Nour [12], Zheng et al. [13], Zhang and Chen [14], and Tschuchnigg et al. [15] have studied the effect of material parameters on the plastic zone of the slope and the strength reduction safety factor. Yang et al. [16] and Chen et al. [17] have studied the range that should be reduced by the strength reduction method. These research achievements are based on the assumption of two strength parameters with the same reduction factor. Nevertheless, concerning the failure process of the slope, the attenuation speed, exerted degree of cohesion, and internal friction angle of the sliding-zone soil are different. Taylor [18] first suggested the adoption of different reduction factors between the cohesion and the internal friction angle and stated that when the slope had a slide, frictional resistance on the sliding surface is fully displayed first and the cohesion can then serve as the supplement. Meanwhile, the safety factor of the slope can be defined as the ratio between the actual cohesion of the sliding surface soil and the cohesion required for display on the sliding surface when the slope can attain the limit equilibrium state. This viewpoint implies that the reduction factor of the internal friction angle is equal to one. Tang et al. $[19,20]$ have suggested the double reduction factors 
method for gradual failure of the soil slope. By changing the proportional relationship between the cohesion reduction factor and the internal friction reduction factor, they studied the reducion principle of the double reduction parameters of the clayey soil slope and sandy soil slope, respectively. The arithmetic mean of the double reduction factors in the limit equilibrium state can be used as the whole safety factor for the slope. Jiang et al. [21] have noted that the proportion between the cohesion reduction factor and the internal friction angle reduction factor for the isotropic soil slope should be 1.75 , and, in the same way, the arithmetic mean of the double reduction factors in the limit equilibrium state can be used as the whole safety factor for the slope. Yuan et al. [22, 23] have studied the proportional relationship between two reduction factors for different slopes in combination with a computational example and have provided two methods of defining the comprehensive safety factor. Isakov et al. [24, 25] have established a formula between the comprehensive safety factor and strength reduction pathway and have suggested an expression formula for the slope minimum comprehensive safety factor using the shortest pathway of strength reduction. Zhao et al. [26] have noted that, for the previously described method, assuming that there is no theoretical basis for the cohesion and the internal friction angle being reduced in terms of a certain proportion, the physical significance of the comprehensive safety factor definition through the shortest pathway of strength reduction is more apparent for the existing comprehensive safety factor definitions. The following can be found from the research on the double reduction factors by the abovementioned scholars: (1) the proportional reduction of the cohesion and friction angle has no theoretical basis and (2) the comprehensive safety factor definition lacks physical significance. Therefore, rationally determining the nonproportional relationship between the double reduction factors and presenting a more rational comprehensive safety factor have become urgent questions to answer.

For the case in which the strength parameter distribution obeys the linear attenuation assumption, a nonproportional relationship between the cohesion reduction factor and the internal friction angle reduction factor is established. By introducing the correlation into the traditional strength reduction finite element method, the nonproportional correlative reduction finite element method for slope strength parameters is proposed. To verify the reliability of the nonproportional correlative reduction finite element method for slope strength parameters, the nonproportional relationship between the cohesion reduction factor and the internal friction angle reduction factor is introduced into Bishop's slice method, yielding the nonproportional double safety factors of Bishop's slice method. For the whole safety storage of the slope based on the double safety factors, a comprehensive safety factor with the contribution of the shear strength parameter to the sliding resistance force as weight is proposed. Finally, in combination with a computational example, the finite element method and Bishop's slice method are adopted, respectively. The parameters between $c$ and $\varphi$ are reduced in terms of the same reduction factor, $c$ is reduced but $\varphi$ is not reduced (i.e., Taylor's frictional circle analysis method implies the reduction way), and the parameters between $c$ and $\varphi$ are reduced in terms of the nonproportional relationship for three types of reduction ways corresponding to the slope stability analysis; results are compared and analyzed to verify the rationality of the nonproportional correlative reduction method. The comparison and analysis of the computational results for the existing comprehensive safety factors indicate that the comprehensive safety factor proposed in this paper has clear physical significance and has a strong practical value.

\section{Basic Principle of the Nonproportional Correlative Reduction Finite Element Method for Slope Strength Parameters}

2.1. Definition of the Slope Double Reduction Factors. In the traditional strength reduction finite element method, the same reduction factor is considered for the cohesion and internal friction angle. Nevertheless, several researchers [1826] have found that, in the failure process of the slope, the functions and their exerted degrees of the shear strength parameters between $c$ and $\varphi$ are indeterminately the same. Accordingly, two strength parameters are naturally considered to have different reduction factors, and the detailed expression formulas are shown in

$$
\begin{aligned}
& \operatorname{SRF}_{c}=\frac{c_{0}}{c}, \\
& \operatorname{SRF}_{\varphi}=\frac{\tan \varphi_{0}}{\tan \varphi},
\end{aligned}
$$

where $c_{0}$ and $\varphi_{0}$ are the initial cohesion and initial internal friction angle, respectively, and $c$ and $\varphi$ are the reduced cohesion and the reduced internal friction angle, respectively. The slope double reduction factors in the limit equilibrium state are the double safety factors. The safety factor of the cohesion can be denoted as $F_{s_{c}}$, and the safety factor of the internal friction angle can be denoted as $F_{s_{\varphi}}$.

\subsection{Basic Assumption of the Strength Parameter Evolution} Laws. The strength parameter evolution laws are generally obtained through indoor and outdoor experiments and numerical testing. If the real strength parameter evolution process can be measured experimentally, then the reduction should be performed in terms of the weakening pathway of the real measured strength parameter. However, for most of the engineering studies, strength parameters such as the peak value time and the residual time are only obtained experimentally. Currently, it is still necessary to determine or propose a set of likely real strength reduction pathways. Therefore, the assumption that there is linear attenuation between the strength parameters at the peak value time and those at the residual time remains in agreement with the simple linear softening model and embodies the correlation between the formulation of shear bands and the softening behaviors of the soil [27]. The simple linear softening model is used to illustrate the nonproportional correlative reduction method for slope strength parameters. Note that the method 


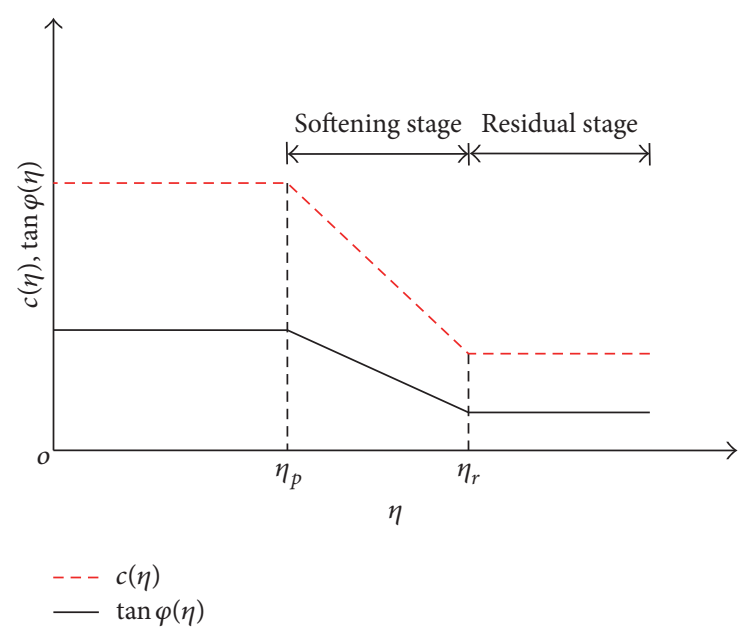

FIGURE 1: Shear strength parameters $c$ and $\varphi$ evolution curve.

proposed in this paper cannot be restricted to the assumed conditions.

Under the simple linear softening model, the strength parameters and the softening parameter $\eta$ are piecewise linear functions [28, 29], as shown in Figure 1, and their formulas are shown in

$$
c(\eta)= \begin{cases}c_{p}, & \left(\eta<\eta_{p}\right), \\ \frac{c_{r}-c_{p}}{\eta_{r}-\eta_{p}}\left(\eta-\eta_{p}\right)+c_{p}, & \left(\eta_{p} \leqslant \eta \leqslant \eta_{r}\right), \\ c_{r}, & \left(\eta>\eta_{r}\right),\end{cases}
$$

$$
\begin{aligned}
& \tan \varphi(\eta) \\
& = \begin{cases}\tan \varphi_{p}, & \left(\eta<\eta_{p}\right), \\
\frac{\tan \varphi_{r}-\tan \varphi_{p}}{\eta_{r}-\eta_{p}}\left(\eta-\eta_{p}\right)+\tan \varphi_{p}, & \left(\eta_{p} \leqslant \eta \leqslant \eta_{r}\right), \\
\tan \varphi_{r}, & \left(\eta>\eta_{r}\right),\end{cases}
\end{aligned}
$$

where $c_{p}, \varphi_{p}$, and $\eta_{p}$ correspond to the cohesion, internal friction angle, and softening parameter at the peak value time, respectively; $c_{r}, \varphi_{r}$, and $\eta_{r}$ correspond to the cohesion, internal friction angle, and softening parameter at the residual time, respectively; and $c(\eta), \varphi(\eta)$, and $\eta$ correspond to the cohesion, internal friction angle, and softening parameter, respectively, at any instant. The softening parameter often uses the equivalent plastic strain or plastic shear strain. The detailed shear stress strength curve varying with the softening parameter is shown in Figure 2.

\subsection{Derivation of the Nonproportional Relationship between} the Double Reduction Factors. From the softening stage formula in (2), the following is obtained:

$$
\frac{\eta-\eta_{p}}{\eta_{r}-\eta_{p}}=\frac{c_{p}-c(\eta)}{c_{p}-c_{r}}
$$

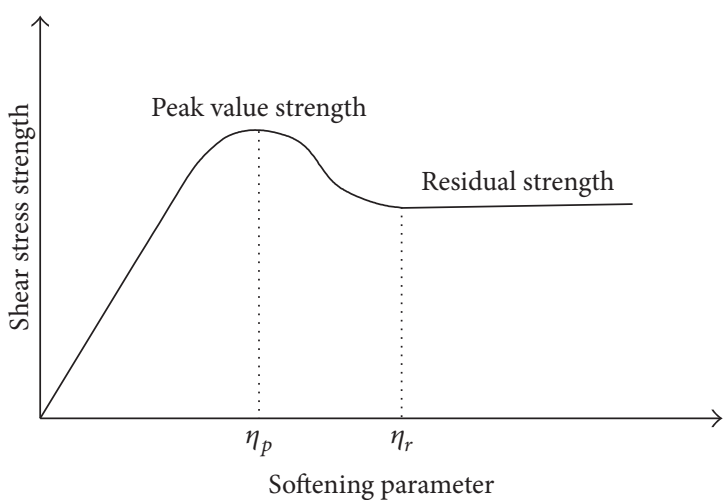

FIGURE 2: Shear stress strength curve varying with the softening parameter.

From the softening stage formula in (3), the following is obtained:

$$
\frac{\eta-\eta_{p}}{\eta_{r}-\eta_{p}}=\frac{\tan \varphi_{p}-\tan \varphi(\eta)}{\tan \varphi_{p}-\tan \varphi_{r}} .
$$

From (4) and (5), the following is obtained:

$$
\frac{c_{p}-c(\eta)}{c_{p}-c_{r}}=\frac{\tan \varphi_{p}-\tan \varphi(\eta)}{\tan \varphi_{p}-\tan \varphi_{r}} .
$$

Substituting (1) into (6), the following is obtained:

$$
\begin{aligned}
\frac{c_{p}\left(\tan \varphi_{p}-\tan \varphi_{r}\right)}{\left(c_{p}-c_{r}\right) \tan \varphi_{p}} & =\frac{\left(\left(\mathrm{SRF}_{\varphi}-1\right) / \mathrm{SRF}_{\varphi}\right)}{\left(\left(\mathrm{SRF}_{c}-1\right) / \mathrm{SRF}_{c}\right)} \\
& =\frac{\operatorname{SRF}_{c}\left(\mathrm{SRF}_{\varphi}-1\right)}{\left(\operatorname{SRF}_{c}-1\right) \mathrm{SRF}_{\varphi}},
\end{aligned}
$$

where $\left(c_{p}-c_{r}\right) / c_{p}$ is denoted as $\lambda_{c}$ and is called the cohesion brittle index, which characterizes the maximum attenuation margin of cohesion in the gradual failure process of the soil. And $\left(\tan \varphi_{p}-\tan \varphi_{r}\right) / \tan \varphi_{p}$ is denoted as $\lambda_{\varphi}$ and is called the friction factor brittle index, which characterizes the maximum attenuation margin of the friction factor in the gradual failure process of the soil.

Thus,

$$
\lambda=\frac{\lambda_{\varphi}}{\lambda_{c}}
$$

is the proportion between the friction factor brittle index and the cohesion brittle index and is called the nonproportional reduction factor. Hence, it is clear that the following expression is true:

$$
\lambda=\frac{c_{p}\left(\tan \varphi_{r}-\tan \varphi_{p}\right)}{\left(c_{r}-c_{p}\right) \tan \varphi_{p}} .
$$

From (7), we obtain the following:

$$
\lambda=\frac{\operatorname{SRF}_{c}\left(\operatorname{SRF}_{\varphi}-1\right)}{\left(\operatorname{SRF}_{c}-1\right) \operatorname{SRF}_{\varphi}} .
$$


From (10), we obtain the following:

$$
\mathrm{SRF}_{\varphi}=\frac{\mathrm{SRF}_{c}}{\mathrm{SRF}_{c}-\left(\mathrm{SRF}_{c}-1\right) \lambda}=\frac{\mathrm{SRF}_{c}}{\lambda+(1-\lambda) \mathrm{SRF}_{c}}
$$

or

$$
\operatorname{SRF}_{c}=\frac{\lambda \mathrm{SRF}_{\varphi}}{1+(\lambda-1) \mathrm{SRF}_{\varphi}} .
$$

When $\lambda=1$, there will be $\mathrm{SRF}_{c}=\mathrm{SRF}_{\varphi}$; that is, we obtain the reduction pathway of the traditional strength reduction finite element method.

The nonproportional relationship between the cohesion reduction factor and the internal friction angle reduction factor is established from the derivation of the above equations. Thus, in the follow-up strength parameter reduction process, the internal friction reduction factor varies as the cohesion reduction factor changes, and the determination of double reduction factors becomes the determination of a single reduction factor. As a result, the blindness of the assumed proportional relationship between the cohesion reduction factor and the internal friction angle reduction factor for the existing double reduction factors method can be overcome. This method can ensure the presence of two states in the process of strength reduction: the peak value state and the residual state. More importantly, the corresponding strength parameters of the two types of states can determine the attenuation rate of the strength parameters. Comparing with traditional strength reduction finite element method in which the two strength parameters are only rigidly attenuated in terms of the same reduction mode, the safety storage in this method is more realistic.

\section{Realization of the Nonproportional Correlative Reduction Finite Element Method for Slope Strength Parameters Based on the Field Variable}

3.1. Computational Principle. The relationships between the cohesion, internal friction angle, and field variable should first be established. The cohesion reduction factor can be defined as the field variable. For the same reason, the internal friction angle reduction factor can also be defined as the field variable. The two types of field variable selection modes have no effect on the computational results for the limit equilibrium state corresponding to a slope. The cohesion must only have an internal friction angle corresponding to it, or an internal friction angle must only have a cohesion corresponding to it. The expression formulas are provided in (13) and (14). Next, we establish the linear relationship between the field variable and the current value of the step time. The formula is given in (15). Hence,

$$
\begin{aligned}
c & =\frac{c_{\text {ini }}}{f}, \\
\varphi & =\arctan \left(\frac{\tan \varphi_{\text {ini }}[\lambda+(1-\lambda) f]}{f}\right), \\
f & =a+b t,
\end{aligned}
$$

where $f$ is the field variable, that is, the cohesion reduction factor $\mathrm{SRF}_{c} ; c_{\text {ini }}$ and $\varphi_{\text {ini }}$ are the cohesion and the internal friction angle, respectively, corresponding to the field variable of one; $t$ is the current value of the step time with $0 \leq$ $t \leq 1$; and $a$ is the initial value of the field variable, whose value has a direct effect on the minimum reduction factor. Therefore, the definition is often less than or equal to one; $b$ can determine the maximum reduction factor of the slope. Therefore, the definition is larger than or equal to three under normal conditions; accordingly, the detailed value of $a$ and $b$ can be conformed through trial computation in terms of computation objectives.

The above descriptions express the rock-soil cohesion and internal friction angle as a function of the field variable and define the field variable as a function of the current value of the step time. For this reason, the reduction of the cohesion and internal friction angle can be automatically realized by increasing the current value of the step time so that the slope reaches the limit equilibrium state. Additionally, the field variable corresponding to this limit equilibrium state is the cohesion safety factor. Substituting the cohesion safety factor into (11), we can obtain the internal friction angle safety factor such that only one computation is required to obtain the safety factor of the slope. Compared with the bisection method, the computational efficiency is improved.

3.2. The Detailed Realization Process. The second development is implemented on the basis of the finite element numerical analysis software ABAQUS. The application of UFIELD has successfully determined the nonproportional correlative reduction finite element method for slope strength parameters based on the field variable and mainly includes the following three steps [30]:

(1) The equilibrium analysis step of the initial geostress should be set up first. The slope should be calculated using the initial shear strength parameters for the stress field under gravity, and the displacement field caused by the initial geostress should be eliminated.

(2) The second analysis step should be set up, that is, the nonproportional correlative reduction analysis step, and the relationship between the material parameters and the field variables can be established. Then, the relationship between the field variables and the current value of the step time should be defined in the UFIELD program. Finally, the accuracy of the reduction factor is controlled using the parameters for the rational setup of the initial increment size, the maximum increment size, and the minimum increment size. Assuming that all of the nodes reach the equilibrium state at increment $i$, for the moment, the analysis step time is $t_{i}$; the flowchart for computing the slope safety factor using the nonproportional correlative reduction finite element method for slope strength parameters is then shown in Figure 3.

(3) The isoline diagram of the equivalent plastic strain obtained from the computation in step (2) should be used to obtain the potential sliding surface [31]. 


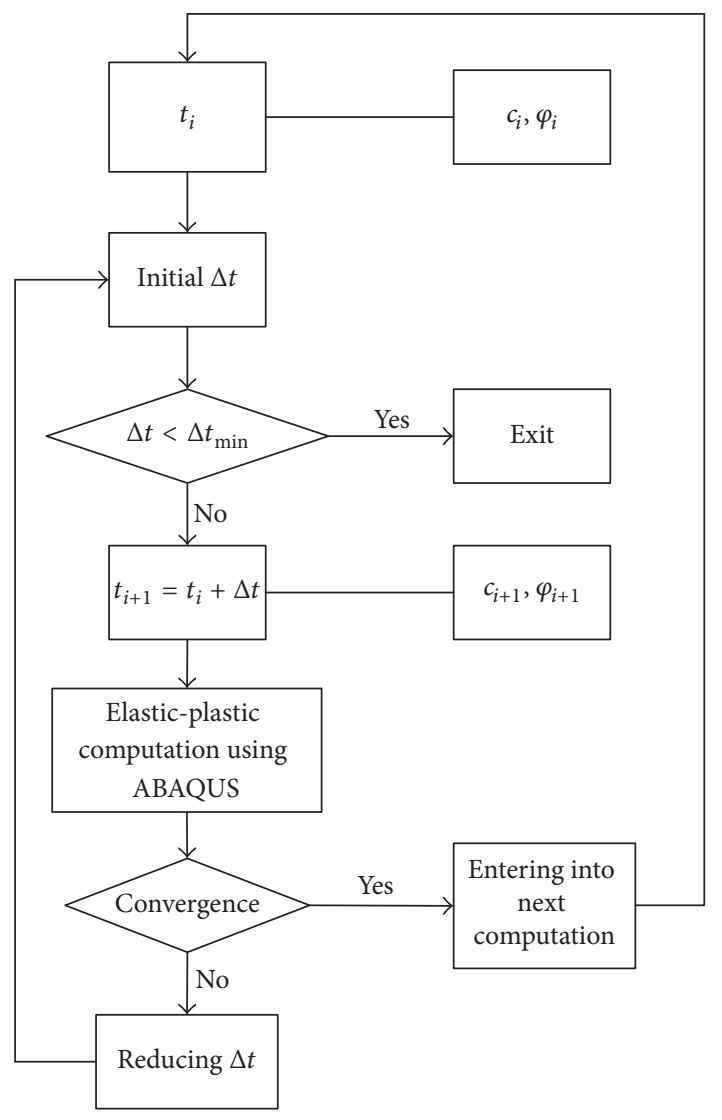

FIGURE 3: Flowchart of the nonproportional correlative reduction method to determine the slope double safety factors.

The cohesion safety factor can be determined from the inflection point of the curve where the horizontal displacement of the characteristic point varies with the cohesion reduction factor. Then, (11) is substituted to obtain the internal friction angle safety factor.

\section{Nonproportional Double Safety Factors of Bishop's Slice Method}

To verify the validity of the nonproportional correlative reduction finite element method for slope strength parameters, the nonproportional relationship between the double safety factors can be introduced into Bishop's slice method, yielding the nonproportional double safety factors based on the limit equilibrium method. The forces acting on the $i$ slice is shown in Figure 4.

As indicated in Figure 4, when the homogeneous soil slope is in the stability state, the shear strength on the sliding surface in any soil slice can only display a part and equilibrium with the tangent force, as follows:

$$
T_{i}=\frac{c_{i} l_{i}}{F_{s_{c}}}+N_{i} \frac{\operatorname{tg} \varphi_{i}}{F_{s_{\varphi}}} .
$$

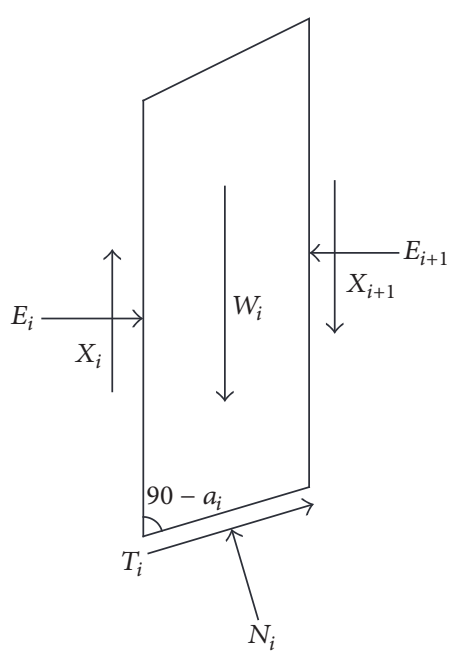

FIGURE 4: Forces acting on the $i$ slice.

In reference to simplified Bishop's slice method, the differences in the friction force acting on the two sides of the slices cannot be accounted for; that is, $X_{i+1}-X_{i}=0$.

For the $i$ soil slice, from $\sum Y=0$, we obtain the following:

$$
W_{i}-N_{i} \cos \alpha_{i}-T_{i} \sin \alpha_{i}=0 .
$$

Substituting (16) into (17), we obtain the following:

$$
N_{i}=\frac{W_{i}-\left(c_{i} l_{i} / F_{s_{c}}\right) \sin \alpha_{i}}{\cos \alpha_{i}+\left(\operatorname{tg} \varphi_{i} / F_{s_{\varphi}}\right) \sin \alpha_{i}} .
$$

When the whole sliding body is in the equilibrium state, the sum of moment acting on the center of the circle of each soil slice should be zero. Therefore, the following expression is obtained:

$$
\sum W_{i} x_{i}-\sum T_{i} R=0
$$

Substituting (16) and (18) into (19), we obtain the following:

$$
\begin{aligned}
& \sum W_{i} \sin \alpha_{i} \\
& \quad=\sum\left(\frac{c_{i} l_{i}}{F_{s_{c}}}+\frac{W_{i}-\left(c_{i} l_{i} / F_{s_{c}}\right) \sin \alpha_{i}}{\cos \alpha_{i}+\left(\operatorname{tg} \varphi_{i} / F_{s_{\varphi}}\right) \sin \alpha_{i}} \frac{\operatorname{tg} \varphi_{i}}{F_{s_{\varphi}}}\right) .
\end{aligned}
$$

Substituting the formula between $F_{s_{c}}$ and $F_{s_{\varphi}}$ in (11) into (20), we obtain the following:

$$
\begin{gathered}
\sum\left(\frac{c_{i} l_{i}}{F_{s_{c}}}+\frac{W_{i}-\left(c_{i} l_{i} / F_{s_{c}}\right) \sin \alpha_{i}}{\cos \alpha_{i}+\left(\operatorname{tg} \varphi_{i}\left(\lambda+(1-\lambda) F_{s_{c}}\right) / F_{s_{c}}\right) \sin \alpha_{i}}\right. \\
\left.\cdot \frac{\operatorname{tg} \varphi_{i}\left(\lambda+(1-\lambda) F_{s_{c}}\right)}{F_{s_{c}}}\right)=\sum W_{i} \sin \alpha_{i} .
\end{gathered}
$$

It can be observed from the above equation that this formula will reduce to only $F_{s_{c}}$ as a variable equation after 
the sliding surface is determined, thus directly obtaining $F_{s_{c}}$. Then, $F_{s_{\varphi}}$ can be computed using (11) such that the two safety factors of the nonproportional double safety factor of Bishop's slice method can be determined. The two safety factors can be renewed continuously using the ongoing changing sliding surface. Finally, the two safety factors corresponding to the most dangerous sliding surface can be obtained; thus, the final double safety factors are determined by the nonproportional double safety factors of Bishop's slice method.

\section{Comprehensive Safety Factor with the Contribution of the Shear Strength Parameter to the Sliding Resistance Force as Weight}

The nonproportional correlative reduction method has decomposed the strength reduction factor into the cohesion reduction factor and the internal friction angle reduction factor. The cohesion safety storage and the internal friction angle safety storage are well described, in terms of the purpose of the experimenters. However, the designers are interested in the comprehensive safety storage of the slope, which is not well solved.

Based on the double safety factors of the slope and to characterize the slope comprehensive safety storage, Tang et al. [19] and Jiang et al. [21] have used the mean value of the double safety factors as the comprehensive safety factor: $F_{s}=\left[F_{s_{c}}+F_{s_{\varphi}}\right] / 2$. Yuan et al. [22, 23] have suggested two types of comprehensive safety factor definitions: $F_{s}=$ $\sqrt{2} F_{s_{c}} F_{s_{\varphi}} / \sqrt{{F_{s_{c}}}^{2}+F_{s_{\varphi}}^{2}}$ and $F_{s}=\sqrt{F_{s_{c}} F_{s_{\varphi}}}$. Isakov et al. [24, 25] have stated that the comprehensive safety factor should be $F_{s}=1 /[1-R / \sqrt{2}]$, where $R=\sqrt{\left(1-1 / F_{s_{c}}\right)^{2}+\left(1-1 / F_{s_{\varphi}}\right)^{2}}$. Accordingly, this paper proposes a comprehensive safety factor that considers each component of the sliding resistance force to the contribution to the sliding resistance force as weight.

In the slope stability analysis, the sliding resistance force consists of three parts. The first part is the contribution of the reinforcement to the sliding resistance force, such as the anchor and slide-resistant pile, denoted as $F_{l}$, and with weight $\omega_{1}$, as shown in (22). The second part is the contribution of the cohesion, with weight $\omega_{c}$, as shown in (23). And the third part is the contribution of the internal friction angle, with weight $\omega_{\varphi}$, as shown in (24). The comprehensive safety factor can be defined as the weighted average of each item's contribution to the sliding resistance force, as shown in (25). Hence,

$$
\begin{gathered}
\omega_{l}=\frac{F_{l}}{F_{l}+\int\left(c_{i} / F_{s_{c}}+\sigma_{i}\left(\tan \varphi_{i} / F_{s_{\varphi}}\right)\right) d s}, \\
\omega_{c}=\frac{\int\left(c_{i} / F_{s_{c}}\right) d s}{F_{l}+\int\left(c_{i} / F_{s_{c}}+\sigma_{i}\left(\tan \varphi_{i} / F_{s_{\varphi}}\right)\right) d s}, \\
\omega_{\varphi}=\frac{\int \sigma_{i}\left(\tan \varphi_{i} / F_{s_{\varphi}}\right) d s}{F_{l}+\int\left(c_{i} / F_{s_{c}}+\sigma_{i}\left(\tan \varphi_{i} / F_{s_{\varphi}}\right)\right) d s},
\end{gathered}
$$

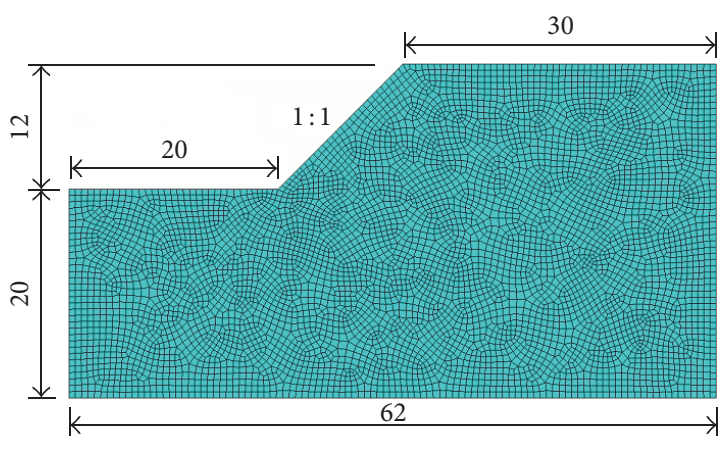

FIgURE 5: Ideal model.

$$
\begin{aligned}
F_{s}= & \omega_{l}+\omega_{c} F_{s_{c}}+\omega_{\varphi} F_{s_{\varphi}} \\
= & \frac{F_{l}}{F_{l}+\int\left(c_{i} / F_{s_{c}}+\sigma_{i}\left(\tan \varphi_{i} / F_{s_{\varphi}}\right)\right) d s} \\
& +\frac{\int c_{i} d s}{F_{l}+\int\left(c_{i} / F_{s_{c}}+\sigma_{i}\left(\tan \varphi_{i} / F_{s_{\varphi}}\right)\right) d s} \\
& +\frac{\int \sigma_{i} \tan \varphi_{i} d s}{F_{l}+\int\left(c_{i} / F_{s_{c}}+\sigma_{i}\left(\tan \varphi_{i} / F_{s_{\varphi}}\right)\right) d s} .
\end{aligned}
$$

It can be easily observed from (25) that the comprehensive safety factor is the ratio between the sliding resistance force actually provided by the soil body and the limit sliding resistance force required to maintain the slope stability, whereas the safety factor obtained using the traditional limit equilibrium method is the percentage or proportion of the sliding resistance force actually provided by the soil body and the sliding force. Accordingly, both are in good agreement. In the case of the nonreinforcement, (25) is simplified to (26). This paper proposes a comprehensive safety factor with the contribution of the shear strength parameter to the sliding resistance force as weight:

$$
F_{s}=\omega_{c} F_{s_{c}}+\omega_{\varphi} F_{s_{\varphi}}=\frac{\int\left(c_{i}+\sigma_{i} \tan \varphi_{i}\right) d s}{\int\left(c_{i} / F_{s_{c}}+\sigma_{i}\left(\tan \varphi_{i} / F_{s_{\varphi}}\right)\right) d s} .
$$

\section{Analysis of a Numerical Example}

6.1. Description of the Finite Element Model and Computational Boundary Conditions. The slope height is $12 \mathrm{~m}$ with a slope ratio of $1: 1$. The distance from the slope toe to the front of the model is $20 \mathrm{~m}$. The distance from the slope crest to the back of the model is $30 \mathrm{~m}$. The total height of the model is $32 \mathrm{~m}$. The total width of the model is $62 \mathrm{~m}$. The computational model can be discretized into 5795 nodes and 5643 elements. The detailed model is shown in Figure 5.

The bottom boundary condition is pinned, and rollers are used along the vertical extents of the finite element mesh.

6.2. Selection of the Computational Parameters. Considering the Mohr-Coulomb failure criterion and the nonassociated 
TABLE 1: Material property parameters used in the computational analysis.

\begin{tabular}{|c|c|c|c|c|c|c|c|}
\hline \multirow[b]{2}{*}{$\begin{array}{l}\text { Density } \\
\left(\mathrm{kg} / \mathrm{m}^{3}\right)\end{array}$} & \multirow[b]{2}{*}{$\begin{array}{l}\text { Elastic modulus } \\
\qquad(\mathrm{MPa})\end{array}$} & \multirow[b]{2}{*}{$\begin{array}{l}\text { Poisson's } \\
\text { ratio }\end{array}$} & \multirow[b]{2}{*}{$\begin{array}{l}\text { Dilation } \\
\text { angle }\left({ }^{\circ}\right)\end{array}$} & \multicolumn{2}{|c|}{ Peak value parameters } & \multicolumn{2}{|c|}{ Residual strength parameters } \\
\hline & & & & $\begin{array}{l}\text { Cohesion } \\
(\mathrm{kPa})\end{array}$ & $\begin{array}{c}\text { Internal } \\
\text { friction angle } \\
\left({ }^{\circ}\right)\end{array}$ & $\begin{array}{l}\text { Cohesion } \\
(\mathrm{kPa})\end{array}$ & Internal friction angle $\left({ }^{\circ}\right)$ \\
\hline 1930 & 56.5 & 0.4 & 0 & 25 & 20 & 7.9 & 16.8 \\
\hline
\end{tabular}
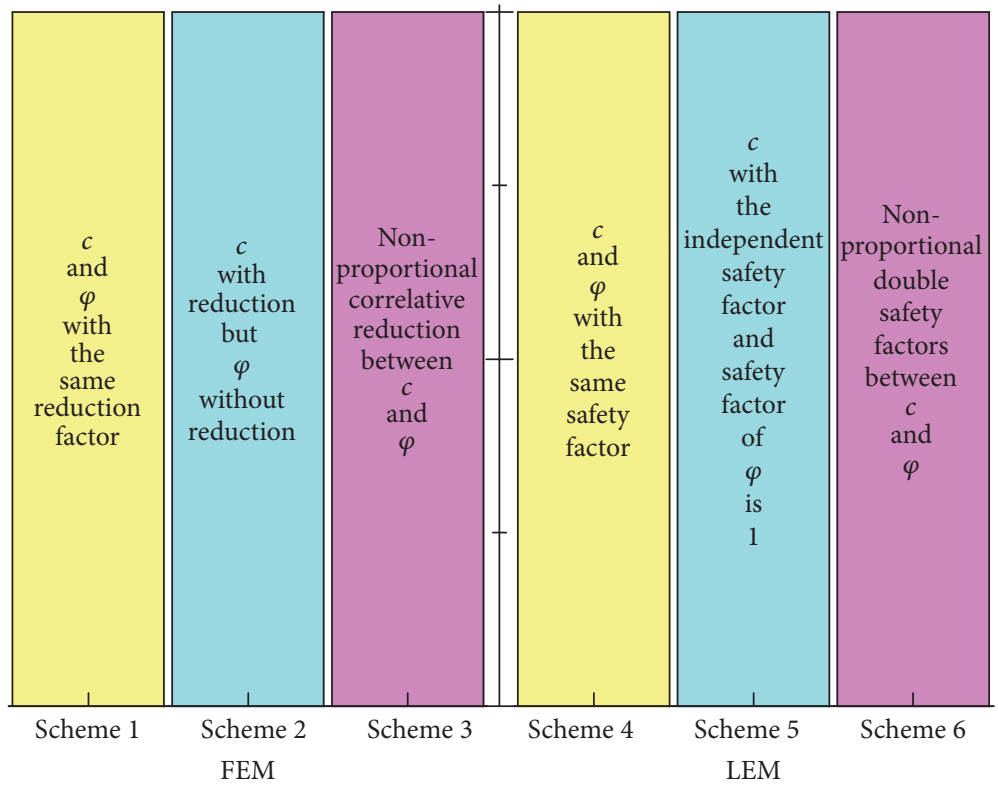

FIGURE 6: Computational schemes corresponding to three types of the reduction modes using FEM and LEM.

flow rule, the ideal elastic-plastic constitutive model is used in the finite element computation. The material property parameters are selected in reference to the experimental results using the predecessors, as shown in Table 1 [32]. The effective coefficients are obtained using the above material property parameters:

$$
\begin{aligned}
\lambda & =\frac{c_{p}\left(\tan \varphi_{p}-\tan \varphi_{r}\right)}{\left(c_{p}-c_{r}\right) \tan \varphi_{p}}=0.249, \\
\operatorname{SRF}_{\varphi} & =\frac{\mathrm{SRF}_{c}}{\lambda+(1-\lambda) \mathrm{SRF}_{c}}=\frac{\mathrm{SRF}_{c}}{0.249+0.751 \times \mathrm{SRF}_{c}} .
\end{aligned}
$$

6.3. Computational Schemes. To discuss the rationality of the nonproportional correlative reduction finite element method for slope strength parameters, a comparative analysis is performed of the results of the slope stability for three types of reduction modes: the reduction modes of the nonproportional correlative reduction finite element method for strength parameters, the traditional strength reduction finite element method, and the method of $c$ with reduction but $\varphi$ without reduction, which is implied in Taylor's friction circle method. A comparative analysis is performed between the finite element method and Bishop's slice method, respectively, corresponding to three types of reduction modes. The detailed computational schemes are shown in Figure 6.

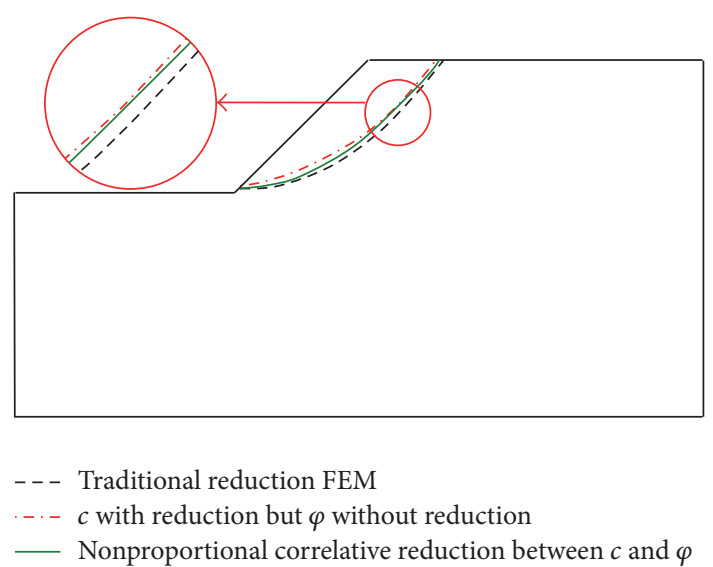

FIGURE 7: Positions of the slope potential sliding surface determined using the different reduction modes.

6.4. Verification of the Reliability of the Computational Results Using the Nonproportional Correlative Reduction Finite Element Method for Slope Strength Parameters. The potential sliding surface in terms of the maximum equivalent plastic strain is shown in Figure 7. It can be observed from Figure 7 that the positions of the slope potential sliding surface obtained using the different reduction modes are different. The positions of the potential sliding surface are close, 
TABLE 2: Computational results for different reduction modes with different methods.

\begin{tabular}{|c|c|c|c|c|}
\hline & & Reduction modes & $\begin{array}{c}\text { Double safety } \\
\text { factors }\end{array}$ & Weight \\
\hline \multirow{6}{*}{$\begin{array}{l}\text { Finite element } \\
\text { method }\end{array}$} & \multirow{2}{*}{$\begin{array}{l}\text { Traditional strength } \\
\text { reduction FEM }\end{array}$} & \multirow{2}{*}{$\begin{array}{l}c \text { and } \varphi \text { with the same reduction factor } \\
\text { (scheme 1) }\end{array}$} & $1.336\left(F_{s_{c}}\right)$ & 0.560 \\
\hline & & & $1.336\left(F_{s_{\varphi}}\right)$ & 0.440 \\
\hline & \multirow{4}{*}{$\begin{array}{l}\text { Double reduction } \\
\text { factors FEM }\end{array}$} & \multirow{2}{*}{$\begin{array}{l}c \text { with reduction but } \varphi \text { without } \\
\text { reduction (scheme } 2 \text { ) }\end{array}$} & $1.717\left(F_{s_{c}}\right)$ & 0.463 \\
\hline & & & $1.000\left(F_{s_{\varphi}}\right)$ & 0.537 \\
\hline & & \multirow{2}{*}{$\begin{array}{l}\text { Nonproportional correlative reduction } \\
\text { between } c \text { and } \varphi \text { (scheme } 3)\end{array}$} & $1.570\left(F_{s_{c}}\right)$ & 0.497 \\
\hline & & & $1.099\left(F_{s_{\varphi}}\right)$ & 0.503 \\
\hline \multirow{6}{*}{$\begin{array}{l}\text { Limit equilibrium } \\
\text { method }\end{array}$} & \multirow{2}{*}{ Bishop's slice method } & \multirow{2}{*}{$\begin{array}{l}c \text { and } \varphi \text { with the same safety factor } \\
\text { (scheme } 4)\end{array}$} & $1.320\left(F_{s_{c}}\right)$ & 0.580 \\
\hline & & & $1.320\left(F_{s_{\varphi}}\right)$ & 0.420 \\
\hline & \multirow{4}{*}{$\begin{array}{c}\text { Double safety factors } \\
\text { of Bishop's slice } \\
\text { method }\end{array}$} & \multirow{2}{*}{$\begin{array}{l}c \text { with the independent safety factor } \\
\text { and safety factor of } \varphi \text { is } 1 \text { (scheme } 5 \text { ) }\end{array}$} & $1.712\left(F_{s_{c}}\right)$ & 0.465 \\
\hline & & & $1.000\left(F_{s_{\varphi}}\right)$ & 0.535 \\
\hline & & \multirow{2}{*}{$\begin{array}{l}\text { Nonproportional double safety factors } \\
\text { between } c \text { and } \varphi \text { (scheme } 6)\end{array}$} & $1.529\left(F_{s_{c}}\right)$ & 0.505 \\
\hline & & & $1.094\left(F_{s_{\varphi}}\right)$ & 0.495 \\
\hline
\end{tabular}

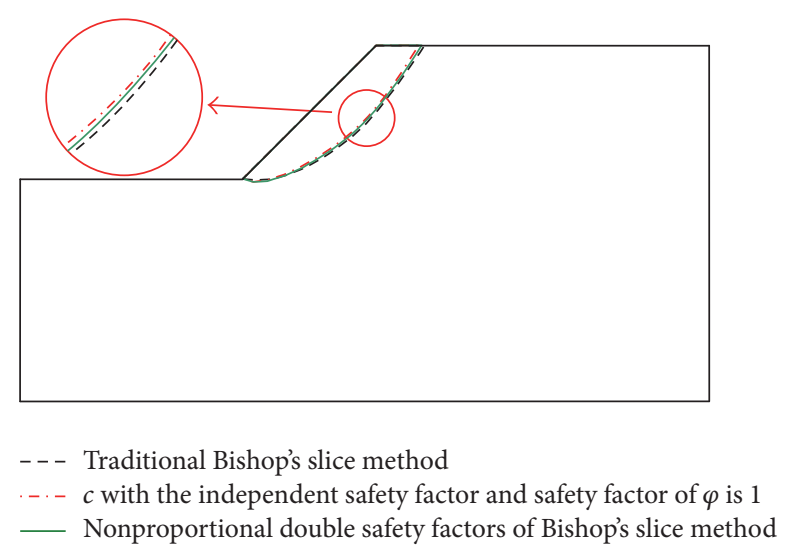

Figure 8: Potential sliding surfaces determined by the double safety factors of Bishop's slice method and traditional Bishop's slice method.

and their morphologies are similar. They are determined using the three types of reduction modes: nonproportional correlative reduction between $c$ and $\varphi, c$ and $\varphi$ reduction in terms of the same reduction factor, and $c$ with reduction but $\varphi$ without reduction. Additionally, the sliding surface determined using the nonproportional correlative reduction method is located between the two sliding surfaces, which are determined by the method between the traditional finite element strength reduction method and $c$ with reduction but $\varphi$ without reduction. The same rules can be found in the positions of the potential sliding surface obtained by the three different types of reduction modes corresponding to Bishop's slice method, as shown in Figure 8.

It can be observed from the computed results that, by adopting the finite element method corresponding to the different reduction modes in Table 2, the slope safety factor determined by the traditional strength reduction finite element method is 1.336 . The slope double safety factors determined by $c$ with reduction and $\varphi$ without reduction are $F_{s_{c}}=1.717$ and $F_{s_{\varphi}}=1.000$, and the weights corresponding to the cohesion and the internal friction angle are 0.463 and 0.537 , respectively. The slope double safety factors determined by the nonproportional correlative reduction method for slope strength parameters are $F_{s_{c}}=1.570$ and $F_{s_{\varphi}}=1.099$, and the weights corresponding to the cohesion and the internal friction angle are 0.497 and 0.503 , respectively. It can be observed from the computed results that, by using Bishop's slice method corresponding to the different reduction modes in Table 2, the slope safety factor determined by Bishop's slice method is 1.320 . The slope double safety factors obtained using $c$ with reduction and $\varphi$ without reduction are $F_{s_{c}}=$ 1.712 and $F_{s_{\varphi}}=1.000$, and the weights corresponding to the cohesion and the internal friction angle are 0.465 and 0.535 , respectively. The double safety factors determined by the nonproportional double safety factors of Bishop's slice method are $F_{s_{c}}=1.529$ and $F_{s_{\varphi}}=1.094$, and the weights corresponding to the cohesion and the internal friction angle are 0.505 and 0.495 , respectively. From the comparative analysis of two types of methods, the computed results for the different reduction modes between Bishop's slice method and finite element method are generally in agreement with each other and illustrate that the results of the finite element method corresponding to the three different types of reduction modes are reliable.

It can be observed from the results of the double safety factors determined by adopting the finite element method that the parameters between $c$ and $\varphi$ produce a reduction of $1.336, \varphi$ without reduction and $c$ produce a reduction of 1.717 , and $c$ produces a reduction of 1.570 and $\varphi$ produces a reduction of 1.099. These can make the slope reach the limit equilibrium state, but this is only possible for the limit equilibrium state obtained through the nonproportional correlative reduction method. The nonproportional correlative reduction method considers the relationship between the material parameter reduction and the deterioration 


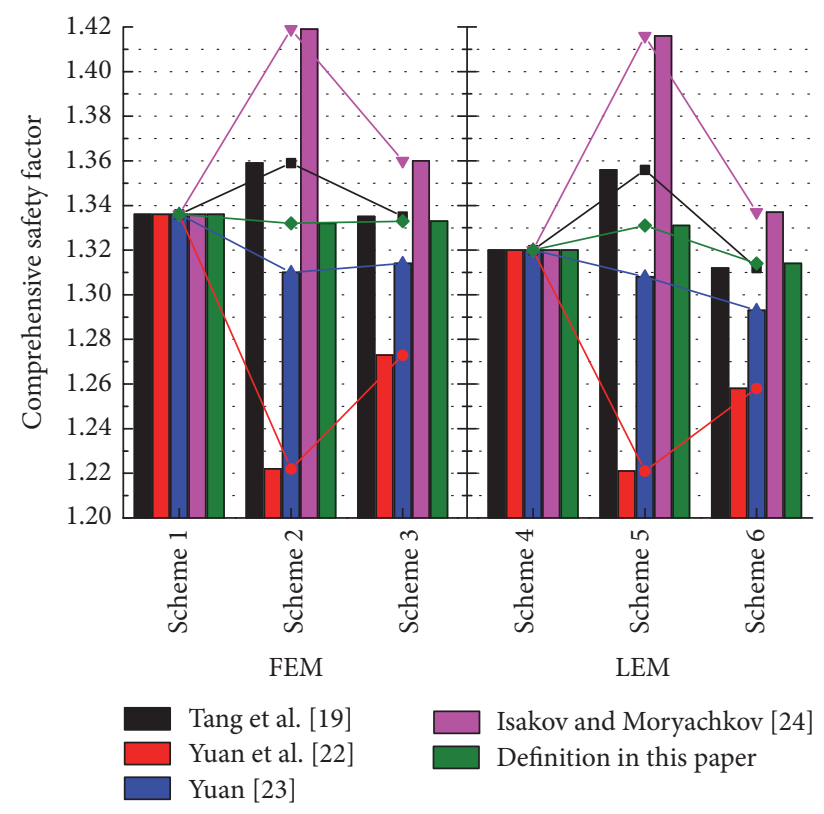

FIGURE 9: Computational results for the comprehensive safety factor from different researches.

of real material properties, whereas the other reduction schemes cannot depend on the real material parameters, they do not model reality, and they correspond to the virtual limit equilibrium states only for easily describing the slope safety factor. Therefore, it is more realistic for the double safety factors to the nonproportional correlative reduction mode.

It can be observed from the comprehensive safety factors of different reduction modes in Figure 9 that there is no considerable difference between the comprehensive safety factors computed by the two types of methods. The maximum difference is 0.023 . The different definitions of the comprehensive safety factor from different researchers reveal that the comprehensive safety factor determined by the nonproportional correlative reduction method lies between the comprehensive safety factor determined by the traditional strength reduction finite element method and the comprehensive safety factor determined by $c$ with reduction but $\varphi$ without reduction. Under the three different types of reduction modes, the computational results of the comprehensive safety factor proposed in this paper are close in value, and the computational results of the comprehensive safety factor corresponding to different reduction modes are close in value to the results of Tang et al. [19] and Yuan [23], illustrating that the comprehensive safety factor proposed in this paper based on the contribution of the shear strength parameter to the sliding resistance force as weight is a rational choice.

Overall, it can observed from the relationships among the results under the three different types of reduction modes that the application of the nonproportional correlative reduction finite element method for strength parameters for analyzing the slope stability is feasible.

\section{Conclusions}

(1) For the example of the strength parameter distribution obeying the linear attenuation assumption, a nonproportional relationship between the cohesion reduction factor and the internal friction angle reduction factor is established.

(2) By introducing a nonproportional correlation into the traditional strength reduction finite element method, the nonproportional correlative reduction finite element method for strength parameters is proposed in this paper.

(3) Using the second development platform of finite element numerical computation software ABAQUS, we realized the nonproportional correlative reduction finite element method for strength parameters based on the field variable, and we improved the efficiency of the solution to the double safety factors of the slope using the finite element method.

(4) To verify the reliability of the nonproportional correlative reduction finite element method for strength parameters, the relationship between the cohesion reduction factor and the internal friction angle reduction factor is introduced into Bishop's slice method, yielding nonproportional double safety factors of Bishop's slice method.

(5) For the whole safety storage of the slope based on the double safety factors, a comprehensive safety factor with the contribution of the shear strength parameter to the sliding resistance force as weight is proposed. This paper can account for the physical significance of the comprehensive safety factor theoretically and illustrate that this comprehensive safety factor is of practical engineering value.

(6) In combination with a computational example and the comparison analysis of the slope sliding surface positions and the comprehensive safety factors for three different types of reduction modes with two types of methods, the rationality and reliability of this method were validated.

\section{Competing Interests}

The authors declare that they have no competing interests.

\section{Acknowledgments}

This study is sponsored by the National Natural Science Foundation of China (no. 51679199), the Key Laboratory for Science and Technology Coordination \& Innovation Projects of Shaanxi Province (no. 2014SZS15-Z01), the Science and Technology Projects of the Transportation Department of Yunnan Province (no. 2014(A) 01), and the Special Funds for Public Industry Research Projects of the Ministry of Water Resources (no. 201501034-04). The financial support provided by these sponsors is greatly appreciated. 


\section{References}

[1] O. C. Zienkiewicz, C. Humpheson, and R. W. Lewis, "Associated and non-associated visco-plasticity and plasticity in soil mechanics," Geotechnique, vol. 25, no. 4, pp. 671-689, 1975.

[2] K. Ugai, "A method of calculation of total safety factor of slope by elasto-plastic FEM," Soils and Foundations, vol. 29, no. 2, pp. 190-195, 1989.

[3] T. Matsui and K.-C. San, "Finite element slope stability analysis by shear strength reduction technique," Soils and Foundations, vol. 32, no. 1, pp. 59-70, 1992.

[4] E. X. Song, "Finite element analysis of safety factor for soil structures," Chinese Journal of Geotechnical Engineering, vol. 19, no. 2, pp. 1-7, 1997.

[5] D. V. Griffiths and P. A. Lane, "Slope stability analysis by finite elements," Geotechnique, vol. 49, no. 3, pp. 387-403, 1999.

[6] E. M. Dawson, W. H. Roth, and A. Drescher, "Slope stability analysis by strength reduction," Geotechnique, vol. 49, no. 6, pp. 835-840, 1999.

[7] J. L. Liu, M. T. Luan, and S. F. Zhao, "Discussion on criteria for evaluating stability of slope in elastoplastic FEM based on shear strength reduction technique," Rock and Soil Mechanics, vol. 26, no. 8, pp. 1345-1348, 2005.

[8] H. Zheng and D.-F. Liu, "Properties of elasto-plastic matrix Dep and a criterion on limiting state of slope stability by FEM," Chinese Journal of Rock Mechanics and Engineering, vol. 24, no. 7, pp. 1099-1105, 2005.

[9] C.-Q. Wu, Y.-W. Zhu, and Y.-Q. Cai, "Dynamic method to assess critical state of slope stability," Rock and Soil Mechanics, vol. 26, no. 5, pp. 784-788, 2005.

[10] L. Y. Zhang, W. M. Shi, and Y. R. Zheng, "The slope stability analysis by FEM under the plane strain condition," Chinese Journal of Geotechnical Engineering, vol. 24, no. 4, pp. 487-490, 2002.

[11] W. X. Fu and Y. Liao, "Non-linear shear strength reduction technique in slope stability calculation," Computers and Geotechnics, vol. 37, no. 3, pp. 288-298, 2010.

[12] M. T. Manzari and M. A. Nour, "Significance of soil dilatancy in slope stability analysis," Journal of Geotechnical and Geoenvironmental Engineering, vol. 126, no. 1, pp. 75-80, 2000.

[13] H. Zheng, D. F. Liu, and C. G. Li, "Slope stability analysis based on elasto-plastic finite element method," International Journal for Numerical Methods in Engineering, vol. 64, no. 14, pp. 18711888, 2005.

[14] P.-W. Zhang and Z.-Y. Chen, "Influences of soil elastic modulus and Poisson's ratio on slope stability," Yantu Lixue/Rock and Soil Mechanics, vol. 27, no. 2, pp. 299-303, 2006.

[15] F. Tschuchnigg, H. F. Schweiger, and S. W. Sloan, "Slope stability analysis by means of finite element limit analysis and finite element strength reduction techniques. Part I: numerical studies considering non-associated plasticity," Computers and Geotechnics, vol. 70, pp. 169-177, 2015.

[16] G. H. Yang, Z. H. Zhong, Y. C. Zhang et al., "Slope stability analysis by local strength reduction method," Rock and Soil Mechanics, vol. 31, no. S2, pp. 53-58, 2010.

[17] G.-Q. Chen, R.-Q. Huang, H. Zhou, Q. Xu, and T.-B. Li, "Research on progressive failure for slope using dynamic strength reduction method," Yantu Lixue/Rock and Soil Mechanics, vol. 34, no. 4, pp. 1140-1146, 2013.

[18] D. W. Taylor, Fundamentals of Soil Mechanics, John Wiley \& Sons, New York, NY, USA, 1948.
[19] F. Tang, Y. R. Zheng, and S. Y. Zhao, "Discussion on two safety factors for progressive failure of soil slope," Chinese Journal of Rock Mechanics and Engineering, vol. 26, no. 7, pp. 1402-1407, 2007.

[20] F. Tang and Y. R. Zheng, "Analysis on safety reserve of slope with two strength reduction factor," Journal of Chongqing Jiaotong University (Natural Science), vol. 26, no. 4, pp. 95-100, 2007.

[21] X. Y. Jiang, Z. G. Wang, L. Y. Liu, and Z. Zhou, "The determination of reduction ratio factor in homogeneous soil-slope with finite element double strength reduction method," The Open Civil Engineering Journal, vol. 7, no. 1, pp. 205-209, 2013.

[22] W. Yuan, B. Bai, X.-C. Li, and H.-B. Wang, "A strength reduction method based on double reduction parameters and its application," Journal of Central South University, vol. 20, no. 9, pp. 2555-2562, 2013.

[23] W. Yuan, Study on Strength Reduction Method, Institute of Rock and Soil Mechanics, Chinese Academy of Sciences, Wuhan, China, 2014.

[24] A. Isakov and Y. Moryachkov, "Estimation of slope stability using two-parameter criterion of stability," International Journal of Geomechanics, vol. 14, no. 3, Article ID 06014004, 2014.

[25] A. L. Isakov, D. A. Korneyev, and Y. Moryachkov, "Twoparameter criterion of road bed stability," in Proceedings of the Engineering Geology, Soil Mechanics, and Foundations, pp. 3138, Siberian State Transport University, Novosibirsk, Russia, 2010.

[26] L.-H. Zhao, J.-Y. Cao, G.-P. Tang, Z.-B. Wang, and H.-H. Tan, "Discussion on slope stability analysis with double strength reduction technique," Yantu Lixue/Rock and Soil Mechanics, vol. 35, no. 10, pp. 2977-2984, 2014.

[27] D.-X. Cheng, D.-A. Liu, E.-B. Ding, X.-Z. Li, and Q.-X. Dai, "Study on attenuation characteristics of long-term strength for landslide soil," Chinese Journal of Rock Mechanics and Engineering, vol. 24, supplement 2, pp. 5827-5834, 2005.

[28] J. P. Carter and S. K. Yeung, "Analysis of cylindrical cavity expansion in a strain weakening material," Computers and Geotechnics, vol. 1, no. 3, pp. 161-180, 1985.

[29] S. Mohammadi and H. A. Taiebat, "A large deformation analysis for the assessment of failure induced deformations of slopes in strain softening materials," Computers and Geotechnics, vol. 49, pp. 279-288, 2013.

[30] Q. J. Xu, H. L. Yin, X. F. Cao, and Z. Li, "A temperaturedriven strength reduction method for slope stability analysis," Mechanics Research Communications, vol. 36, no. 2, pp. 224-231, 2009.

[31] H. Zheng, G. H. Sun, and D. F. Liu, "A practical procedure for searching critical slip surfaces of slopes based on the strength reduction technique," Computers and Geotechnics, vol. 36, no. 1-2, pp. 1-5, 2009.

[32] Y. Q. Ye, Y. C. Zhang, G. H. Yang et al., "Variable modulus elastoplastic strength reduction method application to soil slope stability analysis," Guang Dong Water Resources and Hydropower, no. 7, pp. 4-10, 2013. 


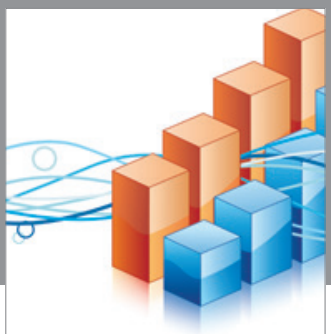

Advances in

Operations Research

vatem alat4

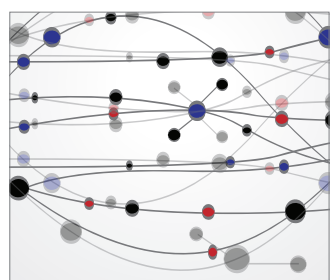

\section{The Scientific} World Journal
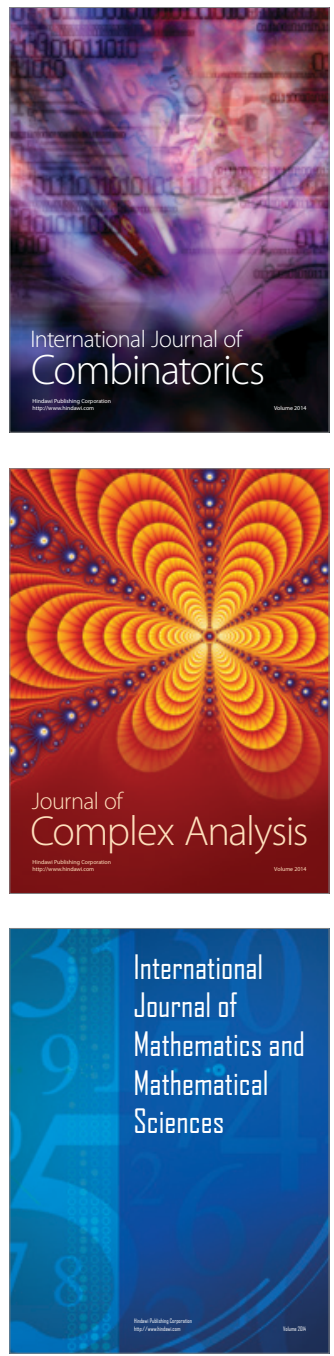
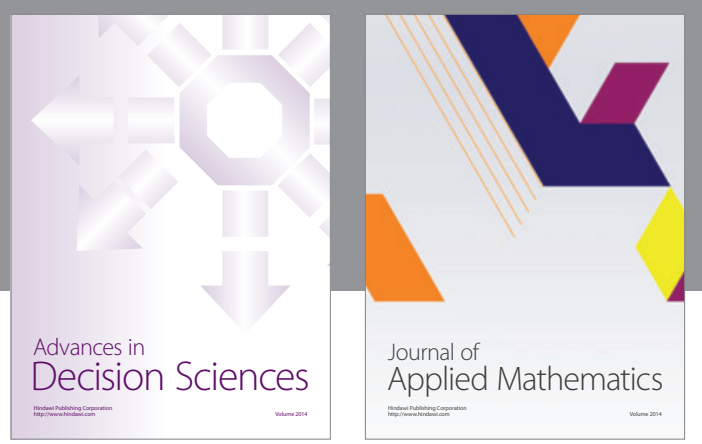

Algebra

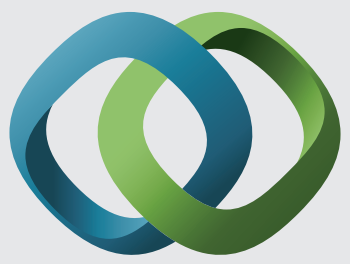

\section{Hindawi}

Submit your manuscripts at

http://www.hindawi.com
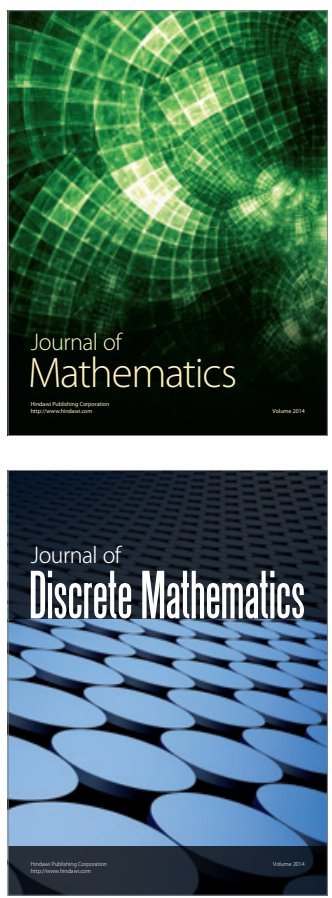

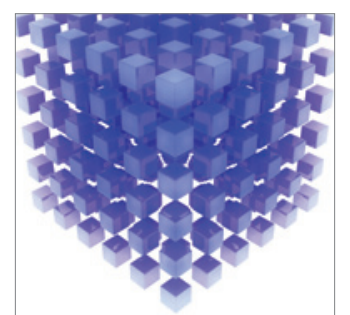

Mathematical Problems in Engineering
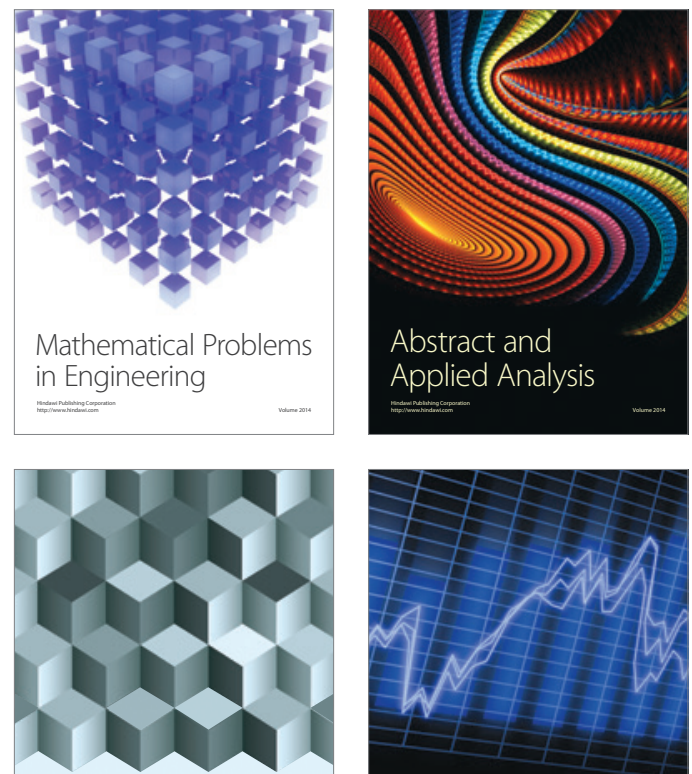

Journal of

Function Spaces

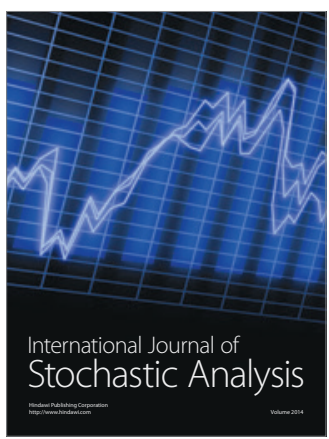

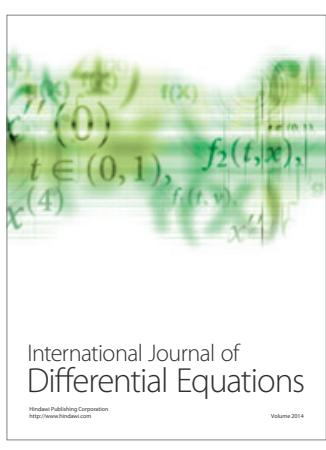
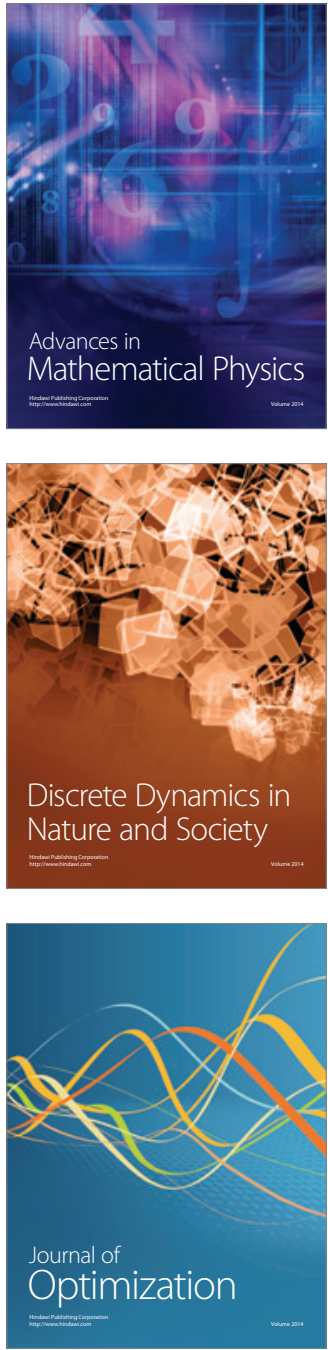